\title{
Platinum Compound
}

National Cancer Institute

\section{Source}

National Cancer Institute. Platinum Compound. NCI Thesaurus. Code C1450.

A chemotherapy compound that contains a platinum atom. Platinum compounds do not contain alkyl groups but do cause DNA crosslinks and adducts similar to alkylating agents. DNA crosslinks and adducts ultimately lead to the induction of apoptosis. 